\title{
Realisierung eines verzerrungsarmen Open-Loop Klasse-D Audio-Verstärkers mit SB-ZePoC
}

\author{
O. Schnick and W. Mathis
}

Institut für Theoretische Elektrotechnik, Leibniz Universität Hannover, Appelstraße 9A, 30167 Hannover, Deutschland

Zusammenfassung. In den letzten Jahren hat die Entwicklung von Klasse-D Verstärkern für Audio-Anwendungen ein vermehrtes Interesse auf sich gezogen. Eine Motivation hierfür liegt in der mit dieser Technik extrem hohen erzielbaren Effizienz von über 90\%. Die Signale, die KlasseD Verstärker steuern, sind binär. Immer mehr Audio-Signale werden entweder digital gespeichert (CD, DVD, MP3) oder digital übermittelt (Internet, DRM, DAB, DVB-T, DVB-S, GMS, UMTS), weshalb eine direkte Umsetzung dieser Daten in ein binäres Steuersignal ohne vorherige konventionelle D/A-Wandlung erstrebenswert erscheint.

Die klassischen Pulsweitenmodulationsverfahren führen zu Aliasing-Komponenten im Audio-Basisband. Diese Verzerrungen können nur durch eine sehr hohe Schaltfrequenz auf ein akzeptables Maß reduziert werden. Durch das von der Forschungsgruppe um Prof. Mathis vorgestellte SB-ZePoC Verfahren (Zero Position Coding with Separated Baseband) wird diese Art der Signalverzerrung durch Generierung eines separierten Basisbands verhindert. Deshalb können auch niedrige Schaltfrequenzen gewählt werden. Dadurch werden nicht nur die Schaltverluste, sondern auch Timing-Verzerrungen verringert, die durch die nichtideale Schaltendstufe verursacht werden. Diese tragen einen großen Anteil zu den gesamten Verzerrungen eines Klasse-D Verstärkers bei. Mit dem SB-ZePoC Verfahren lassen sich verzerrungsarme Open-Loop Klasse-D Audio-Verstärker realisieren, die ohne aufwändige Gegenkopplungsschleifen auskommen.

Abstract. Class-D amplifiers are suiteble for amplification of audio signals. One argument is their high efficiency of $90 \%$ and more. Today most of the audio signals are stored or transmitted in digital form. A digitally controlled Class$\mathrm{D}$ amplifier can be directly driven with coded (modulated)

Correspondence to: O. Schnick

(schnick@ tet.uni-hannover.de) data. No separate D/A conversion is needed. Classical modulation schemes like Pulse-Width-Modulation (PWM) cause aliasing. So a very high switching rate is required to minimize the aliasing component within the signal band. This paper shows a first implementation of the new SB-ZePoC modulation scheme (Zero Position Coding with Separated Baseband), which allows the generation of a binary signal with separated baseband. Therefore Class-D amplifiers using $\mathrm{SB}-\mathrm{ZePoC}$ can be run with very low switching rates. Some benefits and problems in the design process because of low switching rates will be discussed. Measurements of a realtime implementation will be presented.

\section{Einleitung}

Klasse-D Verstärker werden in naher Zukunft eine weite Verbreitung im Audio-Bereich finden (siehe Putzeys, 2003). Die Realisierung eines digital gesteuerten AudioLeistungsverstärkers oder Power-DAC wurde seit den Anfängen der digitalen Audiosignalverarbeitung diskutiert. Die Idee besteht darin, dass digital gespeicherte Daten direkt in ein binäres Steuersignal überführt werden, womit dann ein Schaltverstärker angesteuert werden kann. Digital/AnalogWandlung und Verstärkung werden somit in einem Schritt durchgeführt und der Wirkungsgrad ist aufgrund der KlasseD Technik extrem hoch. Anfangs gab es keine Transistoren auf dem Markt, die den Anforderungen für Full-Range Audioanwendungen gerecht wurden. Als Anfang der 90er Jahre schnelle und leistungsfähige MOSFETs zur Verfügung standen, verhinderten Verzerrungen die bei der Umwandlung von Puls-Code-modulierten (PCM) in Puls-Längen-modulierten (allgemein: PLM, speziell: Puls-Weiten-Modulation PWM) auftreten. 


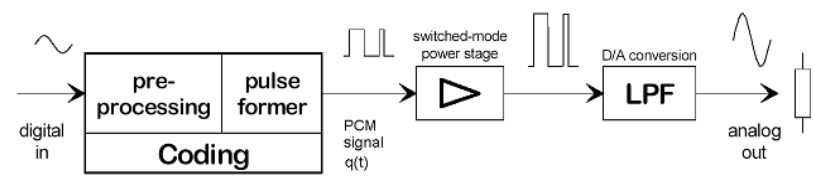

Abb. 1. Blockschaltbild eines Klasse-D Verstärkers.

\section{Digital gesteuerter Klasse-D Verstärker}

Abbildung 1 zeigt das Blockschaltbild eines digital gesteuerten Klasse-D Verstärkers. Die Leistungsschaltendstufe wird mit einem binärem pulslängenmodulierten Signal $q(t)$ betrieben, welches aus einem digitalen Eingangssignal erzeugt wird. Am Ausgang der Schaltendstufe dient ein passives Tiefpassfiler zur Rekonstruktion. Die Generierung des Binärsignals kann mithilfe unterschiedlicher Modulationsbzw. Kodierungsverfahren geschehen. An dieser Stelle sei betont, dass nur das SB-ZePoC (Zero-Position Coding with Separated Baseband) ein separiertes Basisband garantiert, und somit eine exakte Demodulaton mithilfe eines Tiefpassfilters ermöglicht.

\section{Klassische Modulationsverfahren}

Wenn die im PCM-Format gespeicherten Amplitudenwerte einfach in proportionale Pulslängen umgesetzt werden, entspricht das der gleichförmigen Pulsweitenmodulation (UPWM Uniform Pulse Width Modulation). Diese Umsetzung ist mit geringstem Hardwareaufwand möglich, führt allerdings zu erheblichen Signalverzerrungen (Black, 1953): Neben harmonischen Verzerrungen und Intermodulationsverzerrungen treten auch Basisbandverzerrungen (Aliasing) auf, wobei Komponenten des Hochfrequenz-Störspektrums im Übertragungsband erscheinen. Da hier eine digitale Implementierung realisiert werden soll, tritt hier Quantisierungsrauschen aufgrund der endlichen Auflösung des Pulsformes auf. (Black, 1953) zeigt für eine andere Modulationsart, die natürliche Pulsweitenmodulation (NPWM), dass harmonische und Intermodulationverzerrungen eliminiert werden. NPWM setzt aber nicht-äquidistante Abtastpunkte voraus und ist somit für gespeicherte PCM-Audiosignale, die mit einer festen Samplingfrequenz abgetastet wurden, keine geeignete Methode. Die von der Gruppe Goldberg/Sandler eingeführte pseudo-natürliche Pulsweitenmodulation (PNPWM vgl. Goldberg und Sandler, 1991) löst dieses Problem. PNPWM stellt für die digitale Realisierung eine beliebig genaue Näherung von NPWM dar. Das Signal ist also frei von harmonischen und Intermodulationverzerrungen. Es tritt aber weiterhin Aliasing auf. Die einzige Möglichkeit diesen Effekt zu reduzieren besteht in der drastischen Erhöhung der Schaltfrequnz $f_{c}$ des Systems. Die Amplitude der einzelnen Spektrallinien des HF-Störspektrums sinken mit zunehmendem Abstand zur Schaltfrequenz. Die Schaltfrequenz muss

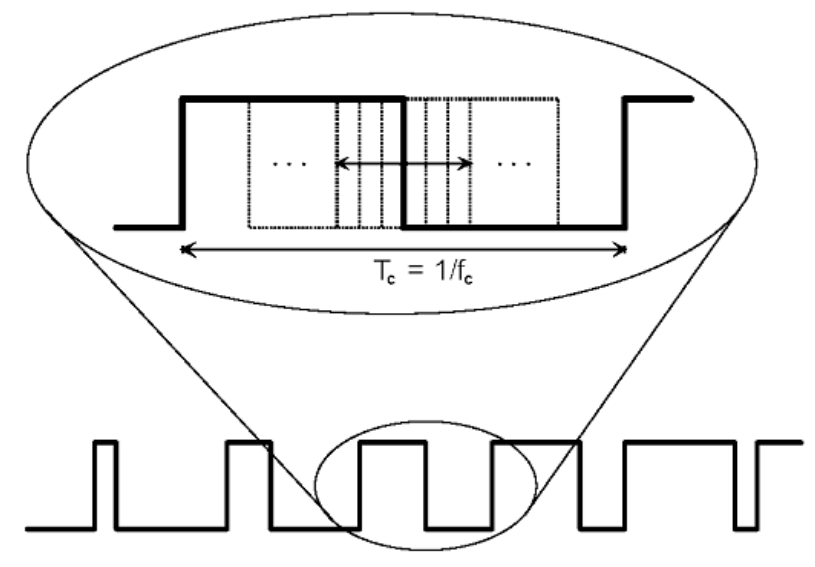

Abb. 2. Binäres Signal $q(t)$.

also soweit erhöht werden, dass die im Basisband auftretenden Komponenten unterhalb eine festgelegten Schwelle liegen. In aktuellen State-Of-The-Art Klasse-D Audioverstärken mit herkömmlichen Modulationverfahren werden bei einem Übertragungsbereich von $20 \mathrm{~Hz}-20 \mathrm{kHz}$ Schaltfrequenzen um $350 \mathrm{kHz}$ verwendet.

\section{Das SB-ZePoC-Verfahren}

Das SB-ZePoC-Verfahren (Streitenberger et al., 2000, 2001; Streitenberger, 2005) basiert auf einem Spektraltheorem, welches Logan bei seiner Arbeit in Zusammenhang mit der Click-Modulation (Logan, 1978) aufstellte. Dieses Verfahren kann mithilfe der digitalen Signalverarbeitung implementiert werden und ist wie PNPWM frei von harmonischen und IMVerzerrungen. Dieses neue Verfahren besitzt aber die zusätzliche Eigenschaft eines separierten Basisbands wie in Abb. 3 gezeigt wird. Die Schaltfrequenz muss nur das doppelte der höchsten Signalfrequenz (als Analogie zum Abtasttheorem von Shannon) plus einer Lücke betragen, die als Guard-Band bezeichnet wird und die flache Tiefpassfilterung mit passiven Bauelementen ermöglicht.

Die Schaltfrequenz eines Systems mit SB-ZePoC kann also erheblich niedriger gewählt werden, als bei den herkömmlichen Modulationsverfahren, ohne dass die Signalqualität beeinträchtigt wird. Die Schaltfrequenz wird somit zum Parameter, der in einem zu tiefen Frequenzen erheblich erweiterten Bereich frei eingestellt werden kann. Die hier gewählte Schaltrate beträgt ca. $100 \mathrm{kHz}$ und ermöglicht eine verzerrungsfreie Signalverstärkung des Audiobands $(20 \mathrm{~Hz}-$ $20 \mathrm{kHz})$.

\section{Design mit niedriger Schaltfrequenz}

Die Möglichkeit eine niedrige Schaltfrequenz wählen zu können hat mehrere Vorteile. Zuerst sind die Schaltverlu- 


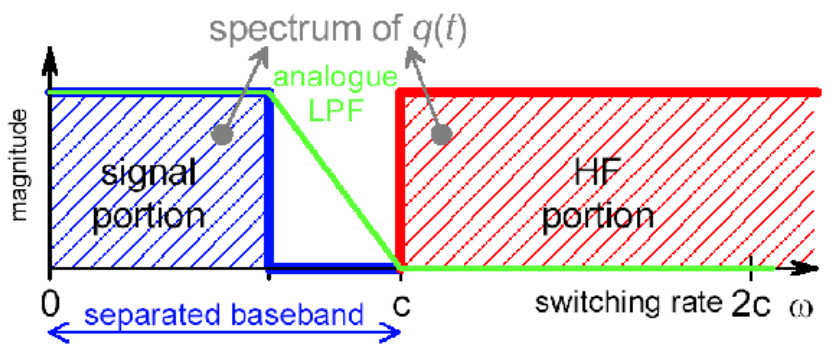

Abb. 3. Spektrum des SB-ZePoC Signals (schematisch).

ste der Transistoren (proportional zur Schaltfrequenz) geringer. Das HF-Störspektrum liegt im Vergleich zu herkömmlichen Systemen in einem niedrigeren Frequenzbereich, wodurch sich Vorteile bei der Elektromagnetischen Verträglichkeit (EMV) ergeben. Die Taktfrequenz des Pulsformers kann niedriger gewählt werden, bzw. bei gleicher Taktfrequenz hat der Pulsformer in einem System mit niedriger Schaltfrequenz eine höhere Auflösung. Der entscheidende Vorteil liegt allerdings in der erzielbaren Signalqualität. Eine der Hauptursachen für Verzerrungen in Klasse-D Verstärkern liegt in den nicht-ideal arbeitenden Leistungsschaltstufen. Bedingt durch die Größe der Schalttransistoren schalten diese nicht unendlich schnell. Um hohe Querströme und damit hohe Verlustleistungen bzw. Zerstörung des Verstärkers zu verhindern, ist es deshalb nötig eine Totzeit zwischen dem oberen und dem unteren Transistor einer Schaltverstärkerbrückenschaltung in das Steuersignal einzufügen. Dadurch werden Verzerrungen im Ausgangsignal verursacht deren, Betrag mithilfe der folgenden Formel abgeschätzt werden kann (Putzeys und de Saint Moulin, 2002):

$T H D_{d}=0.4 \cdot t_{d} \cdot f_{c}$

Die Verzerrungen aufgrund der Totzeit sind näherungsweise direkt proportional zur Schaltfrequenz. Durch die Wahl einer niedrigeren Schaltfrequenz können die Verzerrungen eines Schaltverstärkers ohne Änderung der Hardware reduziert werden. Diese Art der Verzerrung äußert sich qualitativ als Addition ungerader Oberwellen (Mosely et al., 1999; Evans und Close, 1987; Tan et al., 2003; Wu et al., 1999). Bei dem realisierten Verstärker können die Verzerrungen der Schaltendstufe aufgrund der niedrigen Schaltrate von knapp $100 \mathrm{kHz}$ so gering gehalten werden, dass keine Reduzierung mithilfe einer komplizierten Mixed-Signal Gegenkopplungsschleife nötig ist. Der Verstärker kann also als Open-Loop System realisiert werden.

\section{Realisierung}

Die ZePoC-Kodierung wurde als Echtzeitsystem mit fünf Motorola DSPs aufgebaut und ist in Streitenberger (2005) detailliert beschrieben. Zwei FPGAs bilden den Pulsformer.
Die Schaltendstufe wurde als Vollbrückenschaltung mit einstellbarer Totzeit realisiert. Für den Test-Aufbau erfolgt die Spannungsversorgung über Labornetzteile. Da die Zuleitungen ohmsche Verluste aufweisen, werden im Ausgangssignal Verzerrungen verursacht (Risbo und Mørch, 1998). Der Innenwiderstand des Netzteils der Brückenspannung $R_{p s u}$ spielt für die Signalqualität eine entscheidende Rolle. Deren Betrag kann näherungsweise beschrieben werden durch ( $R_{L}$ Lastwiderstand):

$$
T H D=H D_{3}=M^{2} \frac{R_{p s u}}{4 R_{L}} .
$$

Mit $M$ ist hier die Modulationstiefe benannt. Es tritt nur die dritte Harmonische auf. Durch Kapazitäten in der Nähe der Endtransistoren kann der Effekt reduziert werden. Dies gelingt aber nur bis zu einer bestimmten unteren Grenzfrequenz. Bei einem späteren Redesign werden Netzteil und Schaltendstufe räumlich nah beieinander auf demselben Board aufgebaut, so dass der Innenwiderstand des Netzteils sehr niedrig gehalten werden kann und somit der Effekt klein bleibt.

\section{Der Einfluss des Pulsformers}

Das Binärsignal $q(t)$ ist zeitkontinuierlich, kann sich aber nur zu festen diskreten Zeitpunkten ändern (vgl. Abb. 2). Die Takt- oder auch Zählfrequenz des Pulsformers $f_{C L K}$ bestimmt die Größe des minimalen Zeitschrittes $t_{0}$ :

$t_{0}=\frac{1}{f_{C L K}}$.

Die Auflösung des Pulsformers im Zeitbereich in Bit lautet:

$L_{p f}=\log _{2}\left(\frac{f_{C L K}}{f_{c}}\right)$.

Bei einer Schaltfrequenz von $100 \mathrm{kHz}$ und 16 Bit Auflösung müsste die Taktfrequenz $f_{C L K}=6.5536 \mathrm{GHz}$ betragen. Für die Realisierung wurde ein Zähltakt von $200 \mathrm{MHz}$ gewählt. Mit einer Schaltrate von $f_{c}=97.65625 \mathrm{kHz}$ ergibt sich eine Auflösung des Pulsformers von 11 Bit. Es fehlen 5 Bit, wodurch ein ca. $30 \mathrm{~dB}$ schlechterer Signal-Rausch-Abstand (SNR) zu erwarten ist.

\section{Messergebnisse}

Abbildung 4 zeigt das Spektrum der Ausgangsspannung am Lastwiderstand. Die dritte Harmonische bei $-89 \mathrm{~dB}=0.034 \%$ ist die einzig erkennbare Oberwelle. Das SB-ZePoC Signal wird mit einer Genauigkeit von 16 Bit berechnet und vor dem Pulsformer auf 11 Bit gerundet. Weil hierbei kein Dither verwendet wird, ist das Quantisierungsrauschen nicht ganz weiß. Durch Messung mit Zweitonsignalen kann die Menge der auftretenden Quantisierungsfehler 


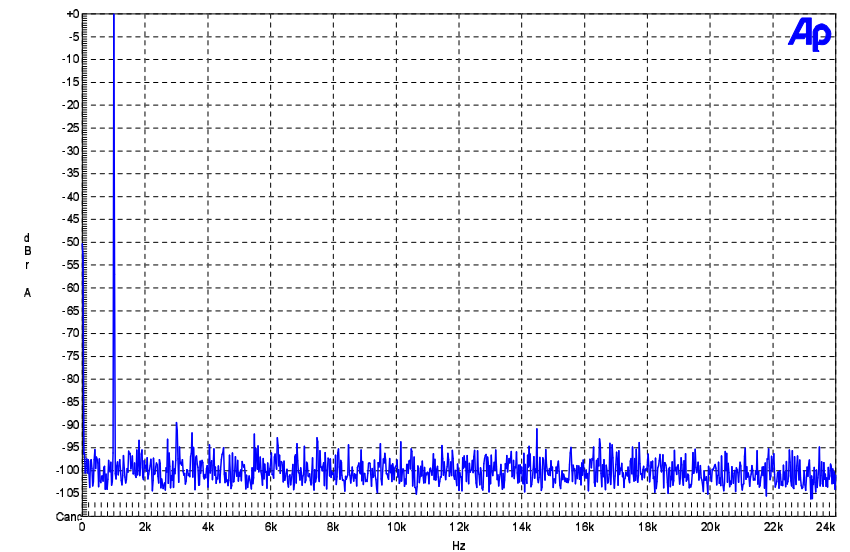

Abb. 4. 32k FFT der Ausgangsspannung bei $10 \mathrm{Veff} 1 \mathrm{kHz}$ an $8 \Omega$ Last bei $0 \mathrm{dBFS}, 0 \mathrm{~dB}$ entspricht der Grundwelle.

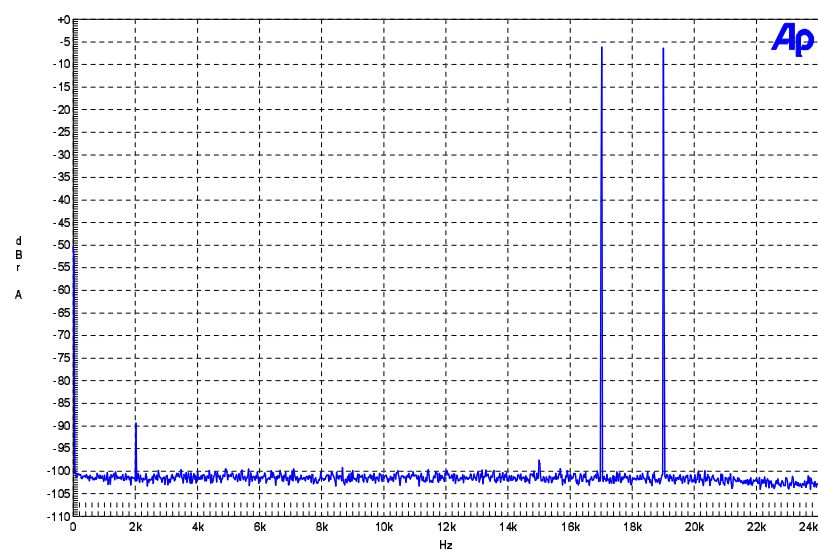

Abb. 5. $32 \mathrm{k}$ FFT Zweitonsignal mit $17 \mathrm{kHz}$ und $19 \mathrm{kHz}$ mit SBZePoC-Verfahren. $0 \mathrm{~dB}$ entspricht 10 Veff an $8 \Omega$.

deutlich erhöht werden. Dadurch erscheint der Frequenzverlauf des Quantisierungsrauschens bei Zweitonsignalen glatter (vgl. Abb. 5, 6 und 7).

Die Abb. 5 und 6 zeigen die Spektren der Ausgangsspannung eines Zweiton-Signals mit Frequenzen von $17 \mathrm{kHz}$ und $19 \mathrm{kHz}$. Es wird das SB-ZePoC-Verfahren dem NPWMVerfahren gegenüber gestellt. In Abb. 6 sind starke Aliasing Komponenten im Basisband zu sehen. Die Schaltfrequenz von rund $100 \mathrm{kHz}$ ist für das PNPWM-Verfahren viel zu niedrig, im Gegensatz zum SB-ZePoC-Verfahren in Abb. 5. Hier sind nur zwei IM-Komponenten bei $15 \mathrm{kHz}$ und bei $2 \mathrm{kHz}$ zu sehen, die hauptsächlich durch den Innenwiderstand des Netzteils verursacht werden. Betrachtet man das Spektrums eines Zweiton-Signals mit $1 \mathrm{kHz}$ und $7 \mathrm{kHz}$, bei dem nur IM-Komponenten hoher Frequenzen auftreten können $(6 \mathrm{kHz}, 8 \mathrm{kHz}, 13 \mathrm{kHz}, 15 \mathrm{kHz}, \ldots)$, liegt die höchste IM-Komponente bei $-96 \mathrm{~dB}$. Dieser Rest an Verzerrungen wird durch die Schaltendstufe verursacht, da in diesem Frequenzbereich die Kompensation des Innenwiderstands durch

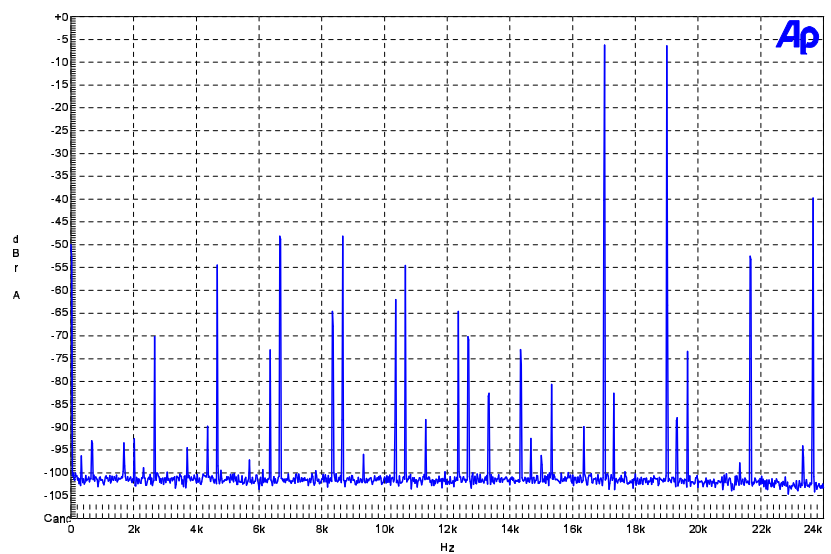

Abb. 6. $32 \mathrm{k}$ FFT Zweitonsignal mit $17 \mathrm{kHz}$ und $19 \mathrm{kHz}$ mit PNPWM-Verfahren. $0 \mathrm{~dB}$ entspricht 10 Veff an $8 \Omega$.

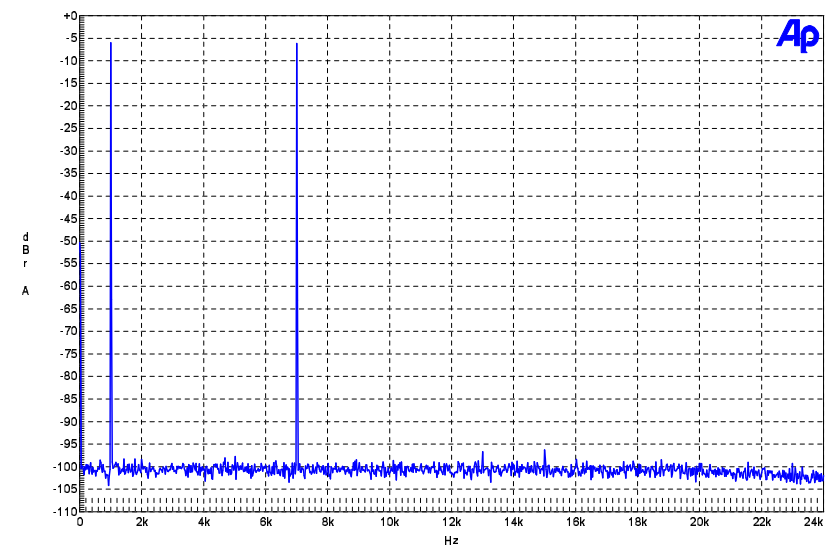

Abb. 7. $32 \mathrm{k}$ FFT Zweitonsignal mit $1 \mathrm{kHz}$ und $7 \mathrm{kHz}$ mit SBZePoC-Verfahren. $0 \mathrm{~dB}$ entspricht 10 Veff an $8 \Omega$.

Kapazitäten sehr effektiv gelingt.

Der Wirkungsgrad der Schaltendstufe erreicht Werte von rund $90 \%$, und weist eine leichte Abhängig vom Lastwiderstand auf. Dieser Wert kann sicherlich noch übertroffen werden, da der Fokus bei dieser Realisierung nicht auf der maximal erreichbaren Effizienz, sondern bei auf möglichst guter Signalqualität lag.

\section{Reduzierung des Quantisierungsrauschens}

Durch die geringe Auflösung des Pulsformers von 11 Bit ist das Quantisierungsrauschen gegenüber der CD $(44,1 \mathrm{kHz}$, 16 Bit) um $30 \mathrm{~dB}$ erhöht. Eine 16 Bit Auflösung würde wie gezeigt einen Pulsformertakt von über $6,5 \mathrm{GHz}$ erfordern. Deshalb muss zur Verringerung des Quantisierungsrauschens ein anderer Weg beschritten werden. In überabgetasteten Systemen bietet sich Rauschformung (im Engl. Noise-Shaping) an. Dabei wird der Quantisierungsrest, der beim Abschnei- 


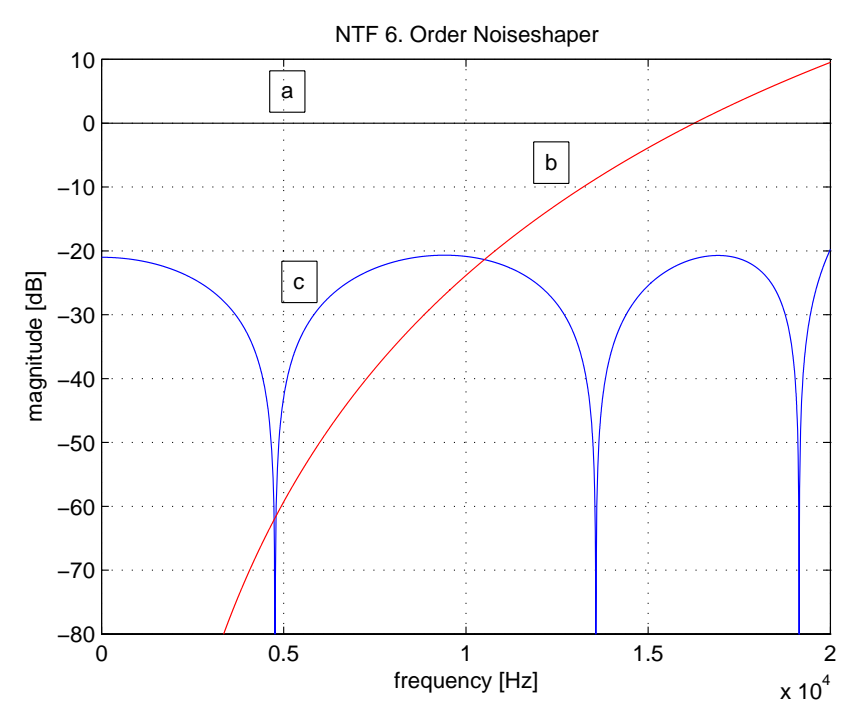

Abb. 8. Vergleich der NTF für Noise-Shaper 6. Ordnung.

den/Runden eines Datenwortes von höherer auf niedrigere Wortlänge entsteht, über ein Filter mit der Übertragungsfunktion $H(z)$ additiv auf den Eingang des Quantisierers zurückgeführt. Für den (Re-)Quantisierungsfehler ergibt sich damit die Rauschübertragungsfunktion $N T F(z)=1-H(z)$. Wird nun für $H(z)$ eine geeignete Tiefpassfunktion gewählt, zeigt $N T F(z)$ Hochpasscharakter. Das Quantisierungsrauschen kann somit im Übertragungsband verringert werden, wobei es oberhalb davon verstärkt wird. Als Ansatz für die Rauschübertragungsfunktion wird oft

$N T F(z)=\left(1-z^{-1}\right)^{N}$.

gewählt. Mit $N$ wird die Ordnung der Rauschformung festgelegt. Bei dieser Rauschübertragungsfunktion liegen alle Nullstellen bei $z=1$ bzw. $f=0$. Da im realisierten System die Überabtastrate (OSR) nur bei etwa 2.5 liegt, wird das Quantisierungsrauschen schon innerhalb des Übertragungsbands (oberhalb von ca. $16 \mathrm{kHz}$ ) verstärkt (vgl. Abb. 8 Kurve b).

Es ist deshalb vorteilhaft, eine Rauschübertragungsfunktion zu wählen, bei der die Nullstellen so über das Übertragungsband verteilt sind, dass das Quantisierungsrauschen im gesamten Übertragungsband reduziert wird (Schreier, 1993)]. Kurve c verdeutlicht dies. Die 0 dB-Linie (Kurve a) stellt zum Vergleich die ideale Rauschübertragungsfunktion für den Fall des reinen Abschneidens/Rundens dar. Das Quantisierungsrauschen wird im gesamten Übertragungsband um mindestens $20 \mathrm{~dB}$ gedämpft. Abbildung 9 zeigt das Ergebnis einer Matlab-Simulation für eine Rauschformung 4. Ordnung.

Da es sich bei der Rauschformung hier um ein rückgekoppeltes System handelt, muss eine Stabilitätsbetrachtung vorgenommen werden. Der (Re-)Quantisierer hat eine Auflösung von 11 Bit. Es kann gezeigt werden, dass die laut

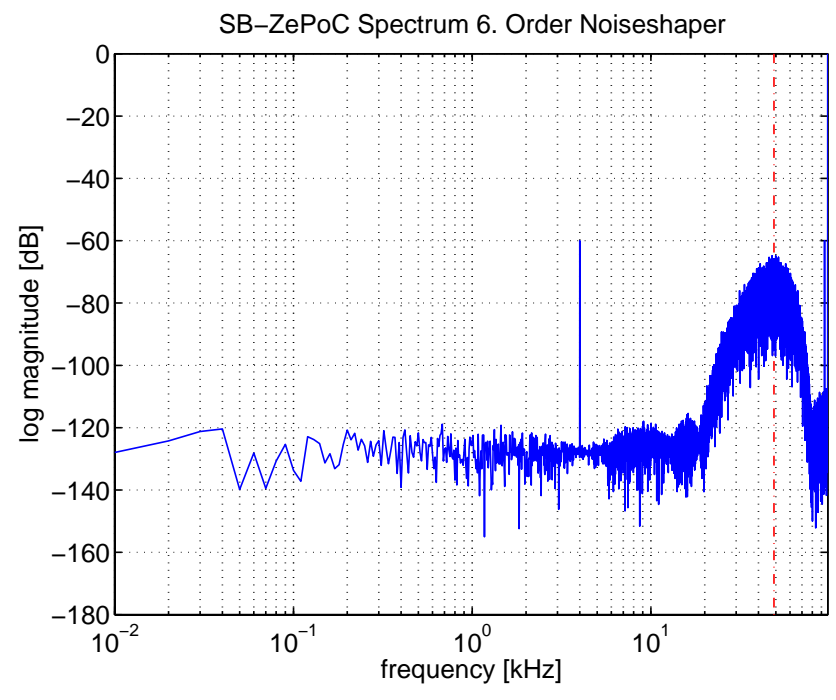

Abb. 9. Matlabsimulation des Systems mit Rauschformung 6. Ordnung.

Schreier und Temes (2004) hinreichende Bedingung für Stabilität

$\max |u(n)| \leq L+2-\|h\|_{1}$

mit

$\|h\|_{1}=\sum_{n=0}^{\infty}|h(n)|$

für Modulationstiefen von $M=0.95$ für Rauschformung bis 8. Ordnung erfüllt ist. $L$ ist die Anzahl der Quantisierungsstufen, hier 2048. Eine Implementierung im System wird demnächst erfolgen. Damit sollte es gelingen, einen KlasseD Verstärker zu realisieren, der die volle CD-Auflösung erreicht.

\section{Zusammenfassung}

Es wurde gezeigt, dass ein mit dem SB-ZePoC-Verfahren digital gesteuerter Open-Loop Klasse-D Audioleistungsverstärker eine gute Performance liefert. Mit diesem Verfahren sind Systeme mit sehr niedrigen Schaltraten im Vergleich zu Systemen mit klassischen Modulationsverfahren möglich. Der realisierte Verstärker erreicht ohne Gegenkopplung sehr gute Verzerrungswerte. Die Messergebnisse werden limitiert durch die aktuelle Auflösung des Pulsformers von 11 Bit. Methoden zur Verringerung des Quantisierungsrauschens im Übertragungsband wurden vorgestellt und $\mathrm{Si}$ mulationsergebnisse präsentiert. Eine Implementierung dieser Methoden wird erfolgen, so dass ein digital gesteuerter Klasse-D Verstärker, der die volle Auflösung der CD $(44.1 \mathrm{kHz}, 16 \mathrm{Bit}$ SNR rund $98 \mathrm{~dB}$ ) erreicht, in naher Zukunft 
realisiert werden kann. Anwendungen für das SB-ZePoCVerfahren sind nicht auf den Audiobereich begrenzt. Die Verwendung sehr niedriger Schaltfrequenzen im Vergleich zu klassischen Verfahren kann z.B. für die Leistungselektronik neue Möglichkeiten eröffnen. Hier werden große und damit langsame Leistungsschalter verwendet wodurch die Höhe der Schaltfrequenz sehr stark eingeschränkt wird.

\section{Literatur}

Putzeys, B.: Digital Audio's Final Frontier - Mini but mighty Class D amps are forging audio's future, IEEE Spectrum, 40, 3, 34-42, March, 2003.

Black, H. S.: Modulation Theory, Van Nostrand, New York, 1953.

Goldberg, J. M. and Sandler, M. B.: Pseudo-natural pulse width modulation for high accuracy digital-to-analogue conversion, Electron. Lett., 27, 16, 1491-1492, 1991.

Streitenberger, M., Felgenhauer, F., Bresch, H., and Mathis, W.: Zero-Position Coding (ZePoC) - A Generalised Concept of Pulse-Length Modulated Signals and its Application to ClassD Audio Power Amplifiers, AES Preprint, No. 5365. 2001, AES 110th Convention, Amsterdam, 12-15 May, 2001.

Streitenberger, M., Felgenhauer, F., and Mathis, W.: Theory and Implementation of a New Type of Digital Power Amplifiers for Audio Applications, Proc. of the IEEE Intern. Symp. on Circuits and Systems, 28-31 May 2000, Geneva, Switzerland (ISCAS2000), I, 511-514, 2000.

Streitenberger, M.: Zur Theorie digitaler Klasse-DSudioleistungsverstärker und deren Implementierung (in German), VDE Verlag GmbH Berlin, 2005.
Logan, Jr., B. F.: Theory of analytic modulation systems, Bell System Technical J., 57, 3, 491-576, 1978.

Putzeys, B., and de Saint Moulin, R.: A True One-Bit Power D/AConverter, presented at the AES 112th convention, Munich, Germany, 11-14 May, 2002.

Mosely, I. D., Mellor, P. H., and Bingham, C. M.: Effect of dead time on harmonic distortion in class-D audio power amplifiers, Electronics Letters, 35, 12, 950-952, 1999.

Evans, P. D. and Close, P. R.: Harmonic distortion in PWM inverter output waveforms, IEE Proc. B, 134, 4, 224-232, 1987.

Tan, M.-T., Chua, H.-C., Gwee, B.-H., and Chang, J. S.: An Investigation on the Paramters Affecting Total Harmonic Distortion in Class D Amplifiers, IEEE Trans. on Circuits and Systems - I, 50, 10, 1304-1315, 2003.

Wu, C. M., Lau, W. H., Chung, H.: Analytical Technique for Calculating the Output Harmonics of an H-Bridge Inverter with Dead Time, IEEE Trans. on Circuits and Systems - I, 46, 5, May, 1999.

Risbo, L., Mørch, T.: Performance of an All-Digital Power Amplification System, 104th Convention of AES, Preprint 4695, May, 1998.

Schreier, R.: An Empirical Study of High-Order Single-Bit DeltaSigma Modulators, IEEE Trans. on Circuits and Systems -0 II, 50, 8, August, 1993.

Schreier, R. and Temes, G.C.: Understanding Delta-Sigma Data Converters, IEEE Press 2004. 